\title{
New Preparation Method of Reynolds' Stain for Transmission Electron Microscope for Liver Samples
}

\author{
Santhana Raj $L^{1^{*}}$, Nur Dayana $M A^{1}$, Norzarila $S N^{1}$, Tan $C V^{2}$ and Nur Afrina $M^{1}$ \\ ${ }^{1}$ Electron Microscopy Unit, Institute for Medical Research, National Institutes of Health, Ministry of Health, \\ Setia Alam, Shah Alam, Malaysia \\ ${ }^{2}$ Epidemiology Unit, Institute for Medical Research, National Institutes of Health, Ministry of Health, Setia \\ Alam, Shah Alam, Malaysia
}

*Corresponding authors: Santhana Raj L, Electron Microscopy Unit, Special Resource Centre, Institute for Medical Research, National Institutes of Health, Jalan Setia Murni U13/52, Seksyen U13 Setia Alam, 40170 Shah Alam, Selangor, Malaysia, Tel: +603-3363-7626

\begin{abstract}
This preparation would maintain good contrast and reduce the formation of lead carbonate in resin-embedded tissue sections. This new method provides a shorter staining protocol of fewer than 5 minutes, extended storage duration, improved contrast, higher broad differential range, and prevents contamination from the environment. Introducing $20 \%$ ethanol to the lead citrate formula makes it to be more stable than lead nitrate formula.
\end{abstract}

\section{Keywords}

Lead citrate, Lead carbonate, Ethanol, Transmission electron microscopy, $\mathrm{NaOH}$

\section{Abbreviations \\ UA: Uranyl Acetate; LN: Lead Nitrate; NLC: New Lead Citrate}

\section{Introduction}

In electron microscopy, images are a magnified projection of various densities of biological structures. Structures that strongly scatter electrons appear as dark areas, while systems that scatter fewer electrons appear bright [1,2]. The most frequently used staining method in TEM is a two-step staining procedure with uranyl acetate (UA), followed by lead citrate [3].

The double contrast method of ultrathin sections on grids with uranyl acetate and lead citrate is the standard routine contrasting technique for electron microscopy.
Although this technique is well known, contrasting is one of the most critical specimen preparation steps for electron microscopy. A standard chemical used is uranyl acetate (UA), which enhances the contrast by interaction with lipids and proteins. However, without using the right concentration, it forms a yellow, needle-like crystal precipitate. Lead citrate works to enhance the contrast by interacting with proteins and glycogens. However, it forms a water-insoluble toxic white precipitate (lead carbonate) if not used strictly under $\mathrm{CO}_{2}$-free conditions [4]. In addition, removing $\mathrm{CO}_{2}$ manually is difficult for which a particular procedure is wanted and need to use to remove $\mathrm{CO}_{2}$.

Lead citrate enhances the contrast effects for a wide range of cellular structures such as ribosomes, lipid membranes, cytoskeleton, and other cytoplasm compartments. The enhancement of the direct effect depends on the interaction with reduced osmium since it allows the attachment of lead ions to the polar groups of molecules. Osmium used as a fixative reacts with UA and lead citrate gives excellent contrast to the samples.

However, detail unpredictable behaviour under laboratory conditions and being time-consuming with delicate preparation are disadvantages in the staining procedure. In addition, lead citrate precipitates readily in the presence of carbon dioxide, either in air or water used for preparing the staining or for rinsing. The 
production of lead carbonate is a water-insoluble toxic white residue and will not dissolve in rinsing water. In general, all lead citrate is harmful to the environment.

This study will show the effects of new lead citrate and standard lead nitrate formulas to observe the contrast, brightness, staining protocol, extended storage duration, and higher broad differential range. It also gives a better understanding of preparing an excellent new Reynold stain to affect studying liver samples' ultrastructure profoundly.

\section{Material and Methods}

We manually stained mice liver tissue samples using the staining protocols of uranyl acetate (UA), lead nitrate $(\mathrm{LN})$ and new lead citrate (NLC). The mice were sourced from Laboratory Animal Resource Unit, Institute for Medical Research. Freshly dissected mouse liver tissues were fixed in $2 \%$ phosphate buffered glutaraldehyde and post-fixed with $2 \%$ osmium tetroxide. Mice liver tissues were dehydrated with $50 \%, 70 \%, 90 \%$ and $100 \%$ (3X) acetone and embedded in Agar 100 epoxy resin and sectioned to a $90 \mathrm{~nm}$ thickness. A sample grid is floated, sectioned side down on the stains following the protocols of UA, then with LN and NLC. After blotting off the stains, the grid was rinsed thoroughly with ultra pure water to remove any residual unbound stain. The stained ultra-thin sections of mouse liver were viewed under TEM (FEI Tecnai G2) at $100 \mathrm{~kW}$.

\section{UA}

1.0 gram of UA (EMS, USA) was dissolved in $100 \mathrm{ml}$ of distilled $\mathrm{H}_{2} \mathrm{O}$ and kept at $8{ }^{\circ} \mathrm{C}$.

\section{LN}

1.33 g lead nitrate (Sigma, USA) mixed in $1.76 \mathrm{~g}$ sodium citrate (Sigma, USA) were shaken vigorously in $30 \mathrm{~mL}$ distilled water. The dissolved solution appeared milky white, and 0.8-gram sodium hydroxide was added and mixed well until the solution turned clear. Finally, the solution was topped up to $50 \mathrm{~mL}$ using distilled water.

\section{NLC}

0.1g lead citrate (Ted Pella, USA) dissolved in 100 $\mathrm{ml}$ of $20 \%$ ethanol. Then, $0.2 \mathrm{~g} \mathrm{NaOH}$ (Sigma, USA) add and mixed well until the solution turned clear and kept at $8{ }^{\circ} \mathrm{C}$.

\section{Results}

Figure 1 and Figure 2 shows the TEM images obtained
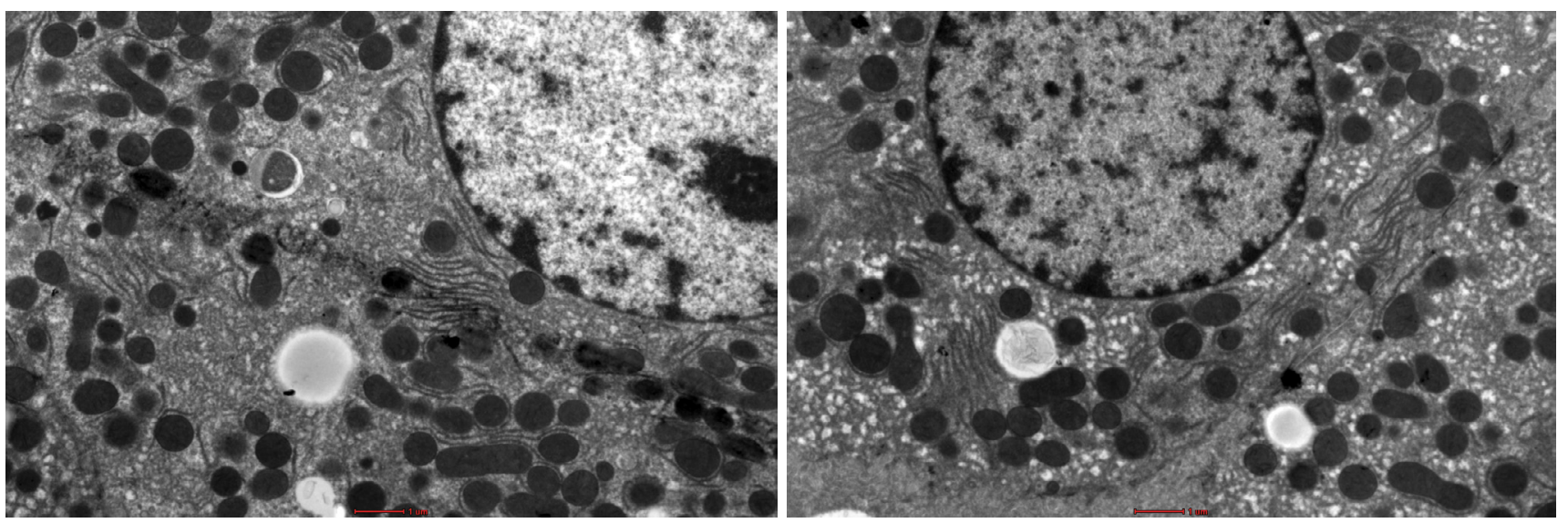

Figure 1: Show variation in the degree of less sharpness, less contrast and less brightness in the stained liver sections using UA and LN. Bar
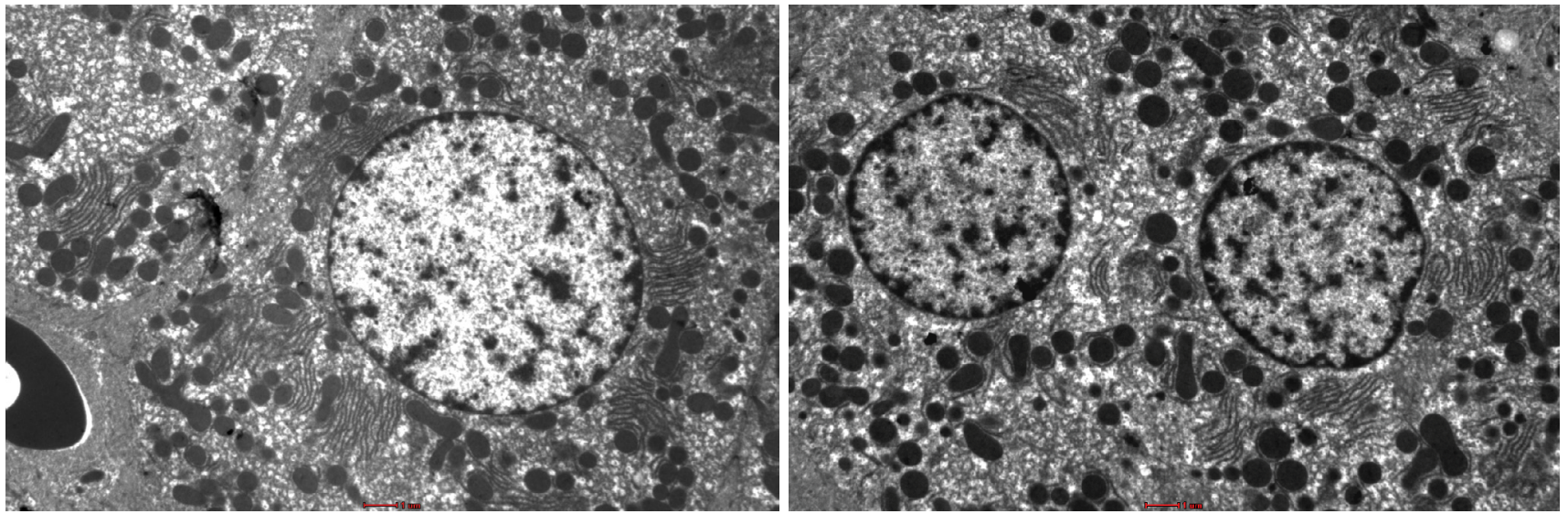

Figure 2: Show variation in the degree of more sharpness, more contrast and more brightness in the stained liver sections using UA and NLC. Bar --------. 
using the different staining protocols. The two images show variation in the degree of sharpness, contrast, and brightness in the stained liver sections. The NLC protocol produced more precise lines of structures as compared to the LN protocol.

\section{Discussion and Conclusion}

The staining protocol in NLC is superior compared with LN. NLC produces quality liver images and provides defined ultrastructure of the kidney's organelles. Moreover, NLC needs a shorter time to complete compared to other staining protocols. NLC staining protocol provides alternative staining to the $\mathrm{LN}$ staining (which is the gold standard in staining electron microscopy ultra-thin sections).

Introducing $20 \%$ ethanol to the formula NLC makes it more robust to environmental contamination. It also makes the brightness and contrasts to be more detailed in the liver samples. Besides that, the effects of osmium tetraoxide on the liver samples also increased and better.

Apart from the liver, other cell cultures and organ tissues such as heart, muscle, and kidney have examined successfully using NLC staining protocol in our laboratory. This NLC staining protocol is safe and faster and can quickly use by other laboratories.

\section{Acknowledgments}

We want to thank the Director-General of Health, Malaysia, for his permission to publish this paper. We also like to extend our gratitude to the Director of the Institute for Medical Research, Malaysia, for supporting this study.

\section{Disclosure}

The author reports no conflicts of interest in this work.

\section{References}

1. Flegler SL, Heckman JW, Klomparens KL (1993) Scanning and transmission electron microscopy: An introduction. Oxford University Press, Inc., New York.

2. Dykstra M (1992) Biological electron microscopy: Theory, techniques, and troubleshooting. Plenum Press, New York and London.

3. Reynolds ES (1963) The use of lead citrate at high pH as an electron-opaque stain in electron microscopy. J Cell Biol 17: $208-212$.

4. Nakakoshi M, Nishioka $H$, Katayama E (2011) New versatile staining reagents for biological transmission electron microscopy that substitute for uranyl acetate. J Electron Microsc (Tokyo) 60: 401-407. 